\title{
Radiomics to better characterize small renal masses
}

Teele Kuusk ${ }^{1,2}$, Joana B. Neves², Maxine Tran'2,3, Axel Bex²,3,4

1. Urology Department, Darent Valley Hospital, Dartford and Gravesham NHS Trust, United Kingdom.

2. Specialist Centre for Kidney Cancer, Royal Free London NHS Foundation Trust, United Kingdom.

3. UCL Division of Surgery and Interventional Science, London, United Kingdom.

4. Surgical Oncology Division, Urology Department, The Netherlands Cancer Institute, The Netherlands

Keywords: renal cell carcinoma, radiomics, small renal mass, imaging, artificial intelligence 


\section{Abstract}

Purpose: Radiomics is a specific field of medical research that uses programmable recognition tools to extract objective information from standard images to combine with clinical data, with the aim of improving diagnostic, prognostic and predictive accuracy beyond standard visual interpretation. We performed a narrative review of radiomic applications that may support improved characterization of small renal masses (SRM). The main focus of the review was to identify and discuss methods which may accurately differentiate benign from malignant renal masses, specifically between renal cell carcinoma (RCC) subtypes and from angiomyolipoma without visible fat (fat-poor AML) and oncocytoma. Further, prediction of grade, sarcomatoid features and gene-mutations would be of importance in terms of potential clinical utility in prognostic stratification and selecting personalised patient management strategies.

Methods: A detailed search of original articles was performed using the PubMed-MEDLINE database until 20 September 2020 to identify English literature relevant to radiomics applications in renal tumour assessment. In total, 42 articles were included in the analysis in 3 main categories related to SRM: prediction of benign versus malignant SRM, subtypes and nuclear grade and other features of aggressiveness.

Conclusion: Overall, studies reported the superiority of radiomics over expert radiological assessment but were mainly of retrospective design and therefore of low quality evidence. However, it is clear that radiomics is an attractive modality that has the potential to improve the non-invasive diagnostic accuracy of SRM imaging and prediction of its natural behaviour. 
Further prospective validation studies of radiomics are needed to augment management algorithms of SRM.

\section{Introduction}

Due to an abundance of quantitative features such as histogram (first-order statistics), texture (gray-level distribution or second-order statistics) and shape present in contemporary cross-sectional imaging of tumours, extracted high-throughput data from Computed Tomography (CT) or Magnetic Resonance Imaging (MRI) scans are used to develop novel radiomic markers for diagnostic, therapeutic and prognostic benefit. Radiomics in renal tumours is an emerging field, and its utility is currently investigated for the characterization of renal masses, distinction of renal cell carcinoma (RCC) subtypes, monitoring response to targeted therapeutic agents and prognosis in the metastatic setting $[1,2]$.

Radiomics is also of special interest to better characterize small renal masses (SRM, defined as renal tumours $\leq 4 \mathrm{~cm}$ diameter), the patient population that is increasing the most. Currently conventional cross-sectional imaging is unable to reliably distinguish between benign and malignant renal tumours despite improvements in qualitative and quantitative imaging techniques $[3,4]$. As a consequence, a retrospective study of 18000 patients undergoing partial nephrectomy in the community setting revealed that more than $30 \%$ of all surgically removed SRM were consistently found to be benign when diagnosis was based on CT alone [5]. Presently, only renal mass biopsy (RMB) has the ability to accurately establish a diagnosis and prevent overtreatment but there are barriers to the uptake of 
$\mathrm{RMB}$ in the urological community, stemming from fear of needle-tract seeding and pathological upstaging as well as diagnostic uncertainties regarding oncocytic tumours $[6,7,3,8,9]$. Nevertheless, avoiding surgery on benign renal lesions as well as tumours of low malignant potential is of paramount importance. A nationwide analysis of renal surgeries over a 4 year period revealed that $3.7 \%$ of resected renal masses were oncocytomas ( $n=1202)[10]$. Of those, only $2.9 \%$ had a preoperative RMB, $43.5 \%$ were SRM and almost half of the patients were 70 years and older suggesting that the majority could have avoided treatment. Altogether, 243 patients (20.2\%) had in-hospital complications of whom 48 had Clavien-Dindo classification grade $\geq \mathrm{III}$ ( $4 \%$ of the total cohort), including three deaths. In addition, strategies of active surveillance (AS) are gaining acceptance, especially in the elderly and comorbid patient population and for tumours of low-malignant potential [1113]. It has recently been shown that growth rate of SRM on AS are subtype and germline $B A P-1$ alteration dependent $[14,15]$. Imaging strategies that could reliably identify benign from malignant renal tumours and predict progression based on aggressive histological or genomic features would enhance an individualised approach to the management of SRM. We reviewed the current available evidence of radiomics and radiogenomics in the context of SRM characterisation.

\section{Methods}

\section{Evidence acquisition}

We performed a detailed search of original articles using the PubMed-MEDLINE database until 20 September 2020 to identify English literature relevant to radiomic applications in renal tumor assessment. We used the search terms 'radiomics', 'artificial intelligence (AI)', 'renal cancer', 'renal cell carcinoma (RCC)', 'kidney cancer', 'renal masses', 'small renal 
masses (SRM)' and 'machine learning (ML)'. In total, 42 articles were included in the narrative review and divided into 3 main categories related to SRM: 1. prediction of benign versus malignant SRM, 2. RCC subtype prediction and 3. prediction of likelihood of progression by nuclear grade, other features of aggressiveness and gene profiles (radiogenomics).

\section{Evidence Synthesis}

\section{Differentiation of benign and malignant renal tumours}

One of the largest retrospective studies involving CT images of 735 patients (539 malignant and 196 benign masses) segmented primary tumours by calculating 33 shape and 760 texture metrics per tumour [16]. Shape features alone achieved an AUC ranging 0.64-0.68 across multiple classifiers, compared with 0.67-0.75 and 0.68-0.75 achieved by texture-only and combined models, respectively. A smaller retrospective study of 79 patients with 84 solid renal masses (63 malignant and 21 benign) extracting 271 texture features from unenhanced and contrast-enhanced CT images demonstrated an AUC of 0.915 for the differentiation between malignant and benign tumours [17] and similar results were described by others $[18,19]$. One machine-learning study specifically addressed the differentiation in cT1 lesions having analysed a total of 94 lesions (76 malignant; 18 benign). The AUC in this small non-validated study reached 0.83 to distinguish malignant from benign tumours with machine-learning [20].

A recent retrospective study of a cohort of 254 RCC (190 clear cell (ccRCC), 38 chromophobe RCCs [chRCC], and 26 papillary RCCs [pRCC]) also included benign lesions such as 26 fat-poor AML, and 10 oncocytomas with preoperative CT examinations [21]. Subsequently, performance values of four expert-level radiologists and the radiomics models were 
compared. Machine-Learning outperformed the radiologists with a sensitivity of $90.0 \%$, $86.3 \%$, and $73.4 \%$ and a specificity of $89.1 \%, 83.3 \%$, and $91.7 \%$, for distinguishing ccRCC from chRCC, subtypes chRCC and cc RCC from the benign AML and oncocytomas and subtypes chRCC and pRCC from AML and oncocytomas, respectively [21].

The largest series using MRI based radiomics included 1162 renal lesions (655 malignant and 507 benign), and compared model performance with expert interpretation and the most optimized radiomics model [22]. Compared with an average of all experts, the best model had higher test accuracy $(0.70$ vs. $0.60, P=0.053)$, sensitivity $(0.92$ vs. $0.80, P=0.017)$, and specificity ( 0.41 vs. $0.35, P=0.450)$.

\section{Differentiation of fat-poor angiomyolipoma (AML) from RCC}

Fat-poor AMLs are often indistinguishable from RCC on contrast enhanced cross sectional imaging. Several studies assessed the discriminative accuracy of different machine learningbased classification models on the differentiation of small $(\leq 4 \mathrm{~cm})$ fat-poor renal $A M L$ and renal cell carcinoma (RCC). In a retrospective analysis of 118 biopsy proven RCC and $45 \mathrm{AML}$ the target region of interest (ROI) was delineated, followed by data extraction of the largest lesion area on each phase of the four-phase CT images [23]. Data were fed into 224 classification models with multiple classifiers resulting in 3360 discriminative models to be analysed for top-ranked features. The highest discriminative models achieved an AUC of 0.90 to distinguish between fat-poor AML and RCC were based on the unenhanced CT phase, alone or in association with images obtained at the nephrographic phase. Features related to shape and to histogram demonstrated superior discrimination compared with texture features [23]. A previous smaller series included 17 and 41 histologically proven fatpoor AML and RCC respectively using three-phase CT images to establish the best 
discriminative classifiers [24]. Overall, several retrospective studies support CT-based radiomics as an non-invasive pre-operative prediction tool for differentiating fat-poor $A M L$ from RCC $[25,24,26-29]$. A. very recent retrospective study suggests that MRI based texture analysis can also be used to distinguish fat-poor AML and oncocytoma from RCC [30]. The authors reported on 54 masses (34 RCC, 14 fat-poor AML and 6 oncocytomas) for which texture parameters on MRI differentiated RCC from fat-poor AML and oncocytoma with AUCs $>0.8$.

\section{Differentiation of RCC subtypes and oncocytoma}

In a restrospective study involving 179 lesions (128 ccRCC and 51 oncocytoma with a mean size of $3.8 \mathrm{~cm}$ (range $0.8-14.6 \mathrm{~cm}$ ) and $3.9 \mathrm{~cm}$ (range 1.0-13.1 cm) respectively, contrastenhanced CT based texture radiomics data collected in the excretory phase predicted oncocytoma with an accuracy of $74.4 \%$, a sensitivity of $85.8 \%$ and a positive predictive value (PPV) of $80.1 \%$ [31]. Another retrospective study of 119 oncocytomas and other RCC subtypes of CT based histogram features demonstrated an excellent AUC of $0.93(p<$ 0.0001), respectively, for differentiating ccRCC from oncocytoma; AUC of $0.99(p<0.0001)$ for differentiating papillary RCC from oncocytoma; and an AUC of 0.92 for differentiating oncocytoma from other subtypes [32]. One study demonstrated differentiation of chromophobe RCC (cRCC) from oncocytoma in 61 patients with confirmed histology (44 CRCC, 17 oncocytomas) using CT based quantitative features with an AUC of 0.85 [33]. 


\section{Differentiation of RCC subtypes \\ Differentiation of RCC subtypes with CT}

CT-based texture radiomics suggest a promising high accuracy to distinguish between ccRCC and other RCC subtypes. In a retrospective study involving 170 patients and a validation cohort of 85 patients the AUC to distinguish between ccRCC and non-ccRCC was 0.949 [34]. In a study including 169 biopsy proven tumours and CT scans texture based radiomics reached AUCs from 0.87 to 0.93 for chromophobe, papillary and ccRCC subtypes [35]. Another retrospective study included 68 RCCs for model development and internal validation while 26 RCCs were included from public databases (The Cancer Genome AtlasTCGA) for independent external validation. 275 texture features were extracted from unenhanced and corticomedullary phase (CMP) CT images [36]. Overall performance of the models derived from CMP images were better than those of unenhanced images. Using CMP discrimination of non-cc-RCCs from cc-RCCs had an external validation accuracy, sensitivity, and specificity of $84.6 \%, 69.2 \%$, and $100 \%$, respectively. However, the performance was poor for distinguishing the three major subtypes and was best for discrimination of pRCC from other RCC subtypes with an external validation accuracy, sensitivity, and specificity of $69.2 \%, 71.4 \%$, and $100 \%$, respectively [36].

\section{Differentiation of RCC subtypes with MRI}

A small study involving 77 tumours with $c C R C C, p R C C$ and chRCC subtypes suggests that radiomic models using shape and texture from three different MRI series has potential to accurately predict subtypes (table 1) [37]. This observation is supported by several other small studies (table 1)[38], the largest one involving 125 patients, 21 of whom with benign tumours but the AUC in the validation cohort of 37 patients ranged from $0.73-0.77$ to 
distinguish between subtypes and benign lesions [39]. Very recently, a study using MRI texture and histogram based models in $90 \mathrm{ccRCC}$ and $22 \mathrm{p} \mathrm{RCCs}$ and 30 oncocytomas showed accuracies of $77.9-79.3 \%$ to distinguish these histologies [40].

\section{Prognosis assessment}

\section{Prediction of sarcomatoid RCC}

In a retrospective study involving 29 sarcomatoid and 99 ccRCC cases, 1029 features were extracted from each of the CMP and nephrographic phase (NP) CT images. The AUC based on the radiomics models for the combined phases was 0.966 suggesting that the presence of sarcomatoid dedifferentiation could potentially be reliably assessed with $\mathrm{Al}$ [41], if validated by other studies.

\section{Prediction of grade in ccRCC}

In a study involving 161 and 99 patients with low and high Fuhrman grade ccRCC 1029 radiomic features were extracted from their CMP and NP CT images [42]. Combining features from both phases, the diagnostic accuracy reached 0.777 with an AUC of 0.822 [95\% Cl: 0.769-0.866; sensitivity, 0.677; specificity, 0.839]. The authors concluded that radiomic features may predict Fuhrman grade [42] as did others in 232 ccRCC [43]. After the grading system changed, the same investigators analysed the predictive value in an expanded group using the same methodology for World Health Organization/International Society of Urologic Pathology (WHO/ISUP) grade differentiation reaching an AUC of 0.9480.98 in the training cohorts with models from both the CMP and NP combined. The AUC remained high in the validation cohort (0.96-0.978) [44]. In two other studies CT textures of 
227 ccRCCs were retrospectively analyzed and revealed an accuracy of more than $90 \%$ predicting the WHO/ISUP grade using corticomedullary (CMP) and parenchyma phase images [45] and an AUC of 0.88 and 0.91 in the training and validation cohort respectively [46].

Recently, the largest retrospective study involving 20 external and 440 internal cases included 322 and $250 \mathrm{MRI}$ and CT texture features for ML-model development to distinguish WHO/ISUP grades [47]. The best MRI and CT based models distinguished high from low WHO/ISUP grade with accuracies of $73 \%$ and $79 \%$. One particular study involving 70 ccRCC showed that MRI based radiomics was superior to tumour size in predicting highgrade disease with AUCs of 0.67 to 0.81 [48].

Another study on 114 patients with resected histologically confirmed ccRCC suggested that texture models based on CT gray-scales can assist in the discrimination of high from low grade ccRCC [49]. The AUC for the models ranged from $0.83-0.88$ but only reached 0.67 in a validation cohort. Smaller studies of 71 and 53 cases of ccRCC suggested that radiomics may be a promising non-invasive technology to distinguish between high and low Fuhrman grades $[50,51]$ (table 2). A recent single centre study in 390 tumours investigated the effects of different methodologies on the performance of $\mathrm{ML}$ models for differentiating high- from low-grade ccRCC [52]. The authors found that models based on limited cropping of CT images, lower complexity and the application of transfer learning achieved the best performance in internal $(A \cup C=0.82 \pm 0.11)$ and external $(A \cup C=0.81 \pm 0.04)$ tests. 


\section{Radio-genomics}

A study in 58 patients of whom 12 developed metastatic disease after surgery used a CT based radiomics model to more accurately predict the postoperative progression of pT1 RCC [53]. In addition, the authors linked radiomics parameters to gene expression profiles generated by whole transcriptome sequencing (WTS). CT based features included histogram and texture. The gene signatures of clinically relevant molecular pathways, tumour immune microenvironment, and potential treatment strategies correlated with the radiomic features which suggests this technique may be used to predict progression in pT1 tumours more accurately and may inform therapeutic options.

Two retrospective studies investigated specifically an association of BAP1 mutation and CT based texture radiomics. One study in $54 \mathrm{RCC}$ and found an AUC of 0.77 to predict BAP1 mutation status [54]. The other study, performed with $65 \mathrm{ccRCC}$ tumours and unenhanced CT based texture radiomics found a sensitivity, specificity, and precision of $90.4 \%, 78.8 \%$, and $81 \%$, respectively for predicting cCRCCs with $B A P-1$ mutation and an AUC of 0.89 [55]. Similarly, another group investigated CT based texture radiomics in predicting the mutation status of the gene encoding the protein polybromo-1 (PBRM1) in 45 patients with ccRCC with a promising AUC of 0.925 [56]. Earlier studies included a retrospective study on 57 ccRCC tumours which reported an AUC 0.85 for VHL, PBRM1 and BAP1 genes [57] and a retrospective study on 78 tumours from the TCGA with an AUC 0f 0.71 based on the nephrogenic phase of the CT to predict BAP1 [58]. 


\section{Evidence interpretation}

The management of SRM would greatly benefit from diagnostic tools indicating which lesions require therapy and which can be safely observed. Currently, only RMB has the potential to accurately characterize SRM but the technique has not been widely adopted. Non-invasive techniques to accurately distinguish benign from malignant lesions and - in case of malignancy - to assess the malignant potential and prognosis of those lesions if left untreated would greatly enhance the management of these patients. Unfortunately, apart from fat-containing AML and homogenous lesions measuring 21-39 HU at portal venousphase, visual interpretation of CT contrast enhanced or MRI cross sectional imaging cannot reliably discern cancer from benign tumours [59].

Given that radiomics mines data from existing resources, it is an attractive potential modality to be explored. Retrospective studies comparing renal tumour histologies suggest a high accuracy and AUC to distinguish benign from malignant tumours. In addition, radiomics harbours potential to predict the course of the disease in case of malignancy. The early results suggesting that $C T$ based radiomics may predict $B A P 1$ mutation status are intriguing in light of the recent publications suggesting higher growth rates of tumours on active surveillance with germline $B A P 1$ alterations. However, the data are too preliminary and it is uncertain if this technique could be exploited to select SRM for active surveillance. This is indicative of a more universal shortcoming of the studies reviewed. None of the multifactorial radiomics algorithms for renal tumours has been translated into routine clinical practice or been independently validated. Two recent systematic review have identified lack of sharing the methodology for data preparation, feature extraction, and poor model construction among the included studies to be responsible for the lack of translation of the proposed algorithms into the clinic $[2,60]$. In addition, reproducibility of 
radiomic features in the very few validation cohorts is questionable and confirmatory studies require larger scale independent and external validation [61]. Another limiting factor of the majority of studies to interpret their findings for the utility in SRM is that they included various tumour sizes. Only one study looked particularly at cT1 lesions and it is uncertain if the radiomic features observed in larger tumours are reproducible in SRM, given the relative inherent tumour heterogeneity in larger tumours.

\section{Future directions}

As several systematic reviews pointed out, a major shortcoming of the current studies to characterize renal masses is their heterogeneity in describing workflow characteristics $[60,61]$. Prospective external and independent validation and diagnostic accuracy studies with reproducible and uniform radiomic features are needed to translate radiomics from research to clinical use.

In conclusion, radiomics to better characterise SRM is an emerging and promising field but not ready for translation into clinical practice. Further sharing of data algorithms, methodology and prospective validation studies are needed if radiomics are to impact on the management of SRM.

\section{Authorś Contribution:}

T Kuusk: Data Collection, Manuscript writing

JB Neves: Data Collection, Manuscript writing

M Tran: Data Collection, Manuscript writing

A Bex: Project development, Data Collection, Manuscript writing

Funding: None

Conflicts of interest: No conflicts of interest 


\section{References:}

1. Lubner MG (2020) Radiomics and Artificial Intelligence for Renal Mass Characterization. Radiol Clin North Am 58 (5):995-1008. doi:10.1016/j.rcl.2020.06.001

2. Ursprung S, Beer L, Bruining A, Woitek R, Stewart GD, Gallagher FA, Sala E (2020) Radiomics of computed tomography and magnetic resonance imaging in renal cell carcinoma-a systematic review and meta-analysis. Eur Radiol 30 (6):3558-3566. doi:10.1007/s00330-020-06666-3

3. Asselin C, Finelli A, Breau RH, Mallick R, Kapoor A, Rendon RA, Tanguay S, Pouliot F, Fairey A, Lavallée LT, Bladou F, Kawakami J, So Al, Richard PO (2020) Does renal tumor biopsies for small renal carcinoma increase the risk of upstaging on final surgery pathology report and the risk of recurrence? Urol Oncol. doi:10.1016/j.urolonc.2020.06.001

4. Schieda N, Lim RS, McInnes MDF, Thomassin I, Renard-Penna R, Tavolaro S, Cornelis FH (2018) Characterization of small $(<4 \mathrm{~cm})$ solid renal masses by computed tomography and magnetic resonance imaging: Current evidence and further development. Diagn Interv Imaging 99 (7-8):443-455. doi:10.1016/j.diii.2018.03.004

5. Kim JH, Li S, Khandwala Y, Chung KJ, Park HK, Chung BI (2019) Association of Prevalence of Benign Pathologic Findings After Partial Nephrectomy With Preoperative Imaging Patterns in the United States From 2007 to 2014. JAMA Surg 154 (3):225-231.

doi:10.1001/jamasurg.2018.4602

6. Marconi L, Dabestani S, Lam TB, Hofmann F, Stewart F, Norrie J, Bex A, Bensalah K, Canfield SE, Hora M, Kuczyk MA, Merseburger AS, Mulders PFA, Powles T, Staehler M, Ljungberg B, Volpe A (2016) Systematic Review and Meta-analysis of Diagnostic Accuracy of Percutaneous Renal Tumour Biopsy. Eur Urol 69 (4):660-673.

doi:10.1016/j.eururo.2015.07.072

7. Herrera-Caceres JO, Finelli A, Jewett MAS (2019) Renal tumor biopsy: indicators, technique, safety, accuracy results, and impact on treatment decision management. World J Urol 37 (3):437-443. doi:10.1007/s00345-018-2373-9

8. Abrahams NA, Tamboli P (2005) Oncocytic renal neoplasms: diagnostic considerations. Clin Lab Med 25 (2):317-339, vi. doi:10.1016/j.cll.2005.01.006

9. Patel HD, Nichols PE, Su ZT, Gupta M, Cheaib JG, Allaf ME, Pierorazio PM (2020) Renal Mass Biopsy is Associated with Reduction in Surgery for Early-Stage Kidney Cancer. Urology 135:76-81. doi:10.1016/j.urology.2019.08.043

10. Neves JB, Withington J, Fowler S, Patki P, Barod R, Mumtaz F, O'Brien T, Aitchison M, Bex A, Tran MGB (2018) Contemporary surgical management of renal oncocytoma: a nation's outcome. BJU Int 121 (6):893-899. doi:10.1111/bju.14159

11. McAlpine K, Breau RH, Stacey D, Knee C, Jewett MAS, Violette PD, Richard PO, Cagiannos I, Morash C, Lavallée LT (2020) Shared decision-making for the management of small renal masses - development and acceptability testing of a novel patient decision aid. Can Urol Assoc J. doi:10.5489/cuaj.6575

12. Goldberg H, Ajaj R, Cáceres JOH, Berlin A, Chandrasekar T, Klaassen Z, Wallis CJD, Ahmad $A E$, Leao R, Petrella AR, Kachura JR, Fleshner N, Matthew A, Finelli A, Jewett MAS, Hamilton RJ (2020) Psychological distress associated with active surveillance in patients younger than 
70 with a small renal mass. Urol Oncol 38 (6):603.e617-603.e625.

doi:10.1016/j.urolonc.2020.02.015

13. Sotimehin AE, Patel HD, Alam R, Gorin MA, Johnson MH, Chang P, Wagner AA, McKiernan JM, Allaf ME, Pierorazio PM (2019) Selecting Patients with Small Renal Masses for Active Surveillance: A Domain Based Score from a Prospective Cohort Study. J Urol 201 (5):886-892. doi:10.1097/ju.0000000000000033

14. Finelli A, Cheung DC, Al-Matar A, Evans AJ, Morash CG, Pautler SE, Siemens DR, Tanguay S, Rendon RA, Gleave ME, Drachenberg DE, Chin JL, Fleshner NE, Haider MA, Kachura JR, Sykes J, Jewett MAS (2020) Small Renal Mass Surveillance: Histology-specific Growth Rates in a Biopsy-characterized Cohort. Eur Urol 78 (3):460-467. doi:10.1016/j.eururo.2020.06.053 15. Ball MW, An JY, Gomella PT, Gautam R, Ricketts CJ, Vocke CD, Schmidt LS, Merino MJ, Srinivasan R, Malayeri AA, Metwalli AR, Linehan WM (2020) Growth Rates of Genetically Defined Renal Tumors: Implications for Active Surveillance and Intervention. J Clin Oncol 38 (11):1146-1153. doi:10.1200/jco.19.02263

16. Yap FY, Varghese BA, Cen SY, Hwang DH, Lei X, Desai B, Lau C, Yang LL, Fullenkamp AJ, Hajian S, Rivas M, Gupta MN, Quinn BD, Aron M, Desai MM, Aron M, Oberai AA, Gill IS, Duddalwar VA (2020) Shape and texture-based radiomics signature on CT effectively discriminates benign from malignant renal masses. Eur Radiol. doi:10.1007/s00330-02007158-0

17. Erdim C, Yardimci AH, Bektas CT, Kocak B, Koca SB, Demir H, Kilickesmez O (2020) Prediction of Benign and Malignant Solid Renal Masses: Machine Learning-Based CT Texture Analysis. Acad Radiol. doi:10.1016/j.acra.2019.12.015

18. Zhang GM, Shi B, Xue HD, Ganeshan B, Sun H, Jin ZY (2019) Can quantitative CT texture analysis be used to differentiate subtypes of renal cell carcinoma? Clin Radiol 74 (4):287294. doi:10.1016/j.crad.2018.11.009

19. Zhou L, Zhang Z, Chen YC, Zhao ZY, Yin XD, Jiang HB (2019) A Deep Learning-Based Radiomics Model for Differentiating Benign and Malignant Renal Tumors. Transl Oncol 12 (2):292-300. doi:10.1016/j.tranon.2018.10.012

20. Uhlig J, Biggemann L, Nietert MM, Beißbarth T, Lotz J, Kim HS, Trojan L, Uhlig A (2020) Discriminating malignant and benign clinical T1 renal masses on computed tomography: $A$ pragmatic radiomics and machine learning approach. Medicine (Baltimore) 99 (16):e19725. doi:10.1097/md.0000000000019725

21. Sun XY, Feng QX, Xu X, Zhang J, Zhu FP, Yang YH, Zhang YD (2020) Radiologic-Radiomic Machine Learning Models for Differentiation of Benign and Malignant Solid Renal Masses: Comparison With Expert-Level Radiologists. AJR Am J Roentgenol 214 (1):W44-w54. doi:10.2214/ajr.19.21617

22. Xi IL, Zhao $Y$, Wang $R$, Chang $M$, Purkayastha $S$, Chang $K$, Huang RY, Silva AC, Vallières $M$, Habibollahi P, Fan Y, Zou B, Gade TP, Zhang PJ, Soulen MC, Zhang Z, Bai HX, Stavropoulos SW (2020) Deep Learning to Distinguish Benign from Malignant Renal Lesions Based on Routine MR Imaging. Clin Cancer Res 26 (8):1944-1952. doi:10.1158/1078-0432.Ccr-19-0374 23. Yang R, Wu J, Sun L, Lai S, Xu Y, Liu X, Ma Y, Zhen X (2020) Radiomics of small renal masses on multiphasic $\mathrm{CT}$ : accuracy of machine learning-based classification models for the differentiation of renal cell carcinoma and angiomyolipoma without visible fat. Eur Radiol 30 (2):1254-1263. doi:10.1007/s00330-019-06384-5

24. Feng Z, Rong P, Cao P, Zhou Q, Zhu W, Yan Z, Liu Q, Wang W (2018) Machine learningbased quantitative texture analysis of $\mathrm{CT}$ images of small renal masses: Differentiation of 
angiomyolipoma without visible fat from renal cell carcinoma. Eur Radiol 28 (4):1625-1633. doi:10.1007/s00330-017-5118-z

25. Nie P, Yang G, Wang Z, Yan L, Miao W, Hao D, Wu J, Zhao Y, Gong A, Cui J, Jia Y, Niu H (2020) A CT-based radiomics nomogram for differentiation of renal angiomyolipoma without visible fat from homogeneous clear cell renal cell carcinoma. Eur Radiol 30 (2):12741284. doi:10.1007/s00330-019-06427-x

26. Lee $H$, Hong $H$, Kim J, Jung DC (2018) Deep feature classification of angiomyolipoma without visible fat and renal cell carcinoma in abdominal contrast-enhanced CT images with texture image patches and hand-crafted feature concatenation. Med Phys 45 (4):1550-1561. doi:10.1002/mp.12828

27. Cui EM, Lin F, Li Q, Li RG, Chen XM, Liu ZS, Long WS (2019) Differentiation of renal angiomyolipoma without visible fat from renal cell carcinoma by machine learning based on whole-tumor computed tomography texture features. Acta Radiol 60 (11):1543-1552. doi:10.1177/0284185119830282

28. Hodgdon T, Mclnnes MD, Schieda N, Flood TA, Lamb L, Thornhill RE (2015) Can Quantitative CT Texture Analysis be Used to Differentiate Fat-poor Renal Angiomyolipoma from Renal Cell Carcinoma on Unenhanced CT Images? Radiology 276 (3):787-796. doi:10.1148/radiol.2015142215

29. Lee HS, Hong H, Jung DC, Park S, Kim J (2017) Differentiation of fat-poor angiomyolipoma from clear cell renal cell carcinoma in contrast-enhanced MDCT images using quantitative feature classification. Med Phys 44 (7):3604-3614. doi:10.1002/mp.12258 30. Razik A, Goyal A, Sharma R, Kandasamy D, Seth A, Das P, Ganeshan B (2020) MR texture analysis in differentiating renal cell carcinoma from lipid-poor angiomyolipoma and oncocytoma. Br J Radiol:20200569. doi:10.1259/bjr.20200569

31. Coy H, Hsieh K, Wu W, Nagarajan MB, Young JR, Douek ML, Brown MS, Scalzo F, Raman SS (2019) Deep learning and radiomics: the utility of Google TensorFlow ${ }^{\mathrm{TM}}$ Inception in classifying clear cell renal cell carcinoma and oncocytoma on multiphasic CT. Abdom Radiol (NY) 44 (6):2009-2020. doi:10.1007/s00261-019-01929-0

32. Yu H, Scalera J, Khalid M, Touret AS, Bloch N, Li B, Qureshi MM, Soto JA, Anderson SW (2017) Texture analysis as a radiomic marker for differentiating renal tumors. Abdom Radiol (NY) 42 (10):2470-2478. doi:10.1007/s00261-017-1144-1

33. Li Y, Huang X, Xia Y, Long $L$ (2020) Value of radiomics in differential diagnosis of chromophobe renal cell carcinoma and renal oncocytoma. Abdom Radiol (NY) 45 (10):31933201. doi:10.1007/s00261-019-02269-9

34. Li ZC, Zhai G, Zhang J, Wang Z, Liu G, Wu GY, Liang D, Zheng H (2019) Differentiation of clear cell and non-clear cell renal cell carcinomas by all-relevant radiomics features from multiphase CT: a VHL mutation perspective. Eur Radiol 29 (8):3996-4007.

doi:10.1007/s00330-018-5872-6

35. Han S, Hwang SI, Lee HJ (2019) The Classification of Renal Cancer in 3-Phase CT Images Using a Deep Learning Method. J Digit Imaging 32 (4):638-643. doi:10.1007/s10278-01900230-2

36. Kocak B, Yardimci AH, Bektas CT, Turkcanoglu MH, Erdim C, Yucetas U, Koca SB, Kilickesmez O (2018) Textural differences between renal cell carcinoma subtypes: Machine learning-based quantitative computed tomography texture analysis with independent external validation. Eur J Radiol 107:149-157. doi:10.1016/j.ejrad.2018.08.014 
37. Wang W, Cao K, Jin S, Zhu X, Ding J, Peng W (2020) Differentiation of renal cell carcinoma subtypes through MRI-based radiomics analysis. Eur Radiol 30 (10):5738-5747. doi:10.1007/s00330-020-06896-5

38. Goyal A, Razik A, Kandasamy D, Seth A, Das P, Ganeshan B, Sharma R (2019) Role of MR texture analysis in histological subtyping and grading of renal cell carcinoma: a preliminary study. Abdom Radiol (NY) 44 (10):3336-3349. doi:10.1007/s00261-019-02122-z

39. Said D, Hectors SJ, Wilck E, Rosen A, Stocker D, Bane O, Beksaç AT, Lewis S, Badani K, Taouli B (2020) Characterization of solid renal neoplasms using MRI-based quantitative radiomics features. Abdom Radiol (NY) 45 (9):2840-2850. doi:10.1007/s00261-020-02540-4 40. Hoang UN, Mojdeh Mirmomen S, Meirelles O, Yao J, Merino M, Metwalli A, Marston Linehan W, Malayeri AA (2018) Assessment of multiphasic contrast-enhanced MR textures in differentiating small renal mass subtypes. Abdom Radiol (NY) 43 (12):3400-3409. doi:10.1007/s00261-018-1625-x

41. Meng X, Shu J, Xia Y, Yang R (2020) A CT-Based Radiomics Approach for the Differential Diagnosis of Sarcomatoid and Clear Cell Renal Cell Carcinoma. Biomed Res Int 2020:7103647. doi:10.1155/2020/7103647

42. Shu J, Tang Y, Cui J, Yang R, Meng X, Cai Z, Zhang J, Xu W, Wen D, Yin H (2018) Clear cell renal cell carcinoma: CT-based radiomics features for the prediction of Fuhrman grade. Eur J Radiol 109:8-12. doi:10.1016/j.ejrad.2018.10.005

43. Lin F, Cui EM, Lei Y, Luo LP (2019) CT-based machine learning model to predict the Fuhrman nuclear grade of clear cell renal cell carcinoma. Abdom Radiol (NY) 44 (7):25282534. doi:10.1007/s00261-019-01992-7

44. Shu J, Wen D, Xi Y, Xia Y, Cai Z, Xu W, Meng X, Liu B, Yin H (2019) Clear cell renal cell carcinoma: Machine learning-based computed tomography radiomics analysis for the prediction of WHO/ISUP grade. Eur J Radiol 121:108738. doi:10.1016/j.ejrad.2019.108738 45. He X, Wei Y, Zhang H, Zhang T, Yuan F, Huang Z, Han F, Song B (2020) Grading of Clear Cell Renal Cell Carcinomas by Using Machine Learning Based on Artificial Neural Networks and Radiomic Signatures Extracted From Multidetector Computed Tomography Images. Acad Radiol 27 (2):157-168. doi:10.1016/j.acra.2019.05.004

46. Sun X, Liu L, Xu K, Li W, Huo Z, Liu H, Shen T, Pan F, Jiang Y, Zhang M (2019) Prediction of ISUP grading of clear cell renal cell carcinoma using support vector machine model based on CT images. Medicine (Baltimore) 98 (14):e15022. doi:10.1097/md.0000000000015022 47. Cui E, Li Z, Ma C, Li Q, Lei Y, Lan Y, Yu J, Zhou Z, Li R, Long W, Lin F (2020) Predicting the ISUP grade of clear cell renal cell carcinoma with multiparametric MR and multiphase CT radiomics. Eur Radiol 30 (5):2912-2921. doi:10.1007/s00330-019-06601-1

48. Dwivedi DK, Xi Y, Kapur P, Madhuranthakam AJ, Lewis MA, Udayakumar D, Rasmussen R, Yuan Q, Bagrodia A, Margulis V, Fulkerson M, Brugarolas J, Cadeddu JA, Pedrosa I (2020) Magnetic Resonance Imaging Radiomics Analyses for Prediction of High-Grade Histology and Necrosis in Clear Cell Renal Cell Carcinoma: Preliminary Experience. Clin Genitourin Cancer. doi:10.1016/j.clgc.2020.05.011

49. Ding J, Xing Z, Jiang Z, Chen J, Pan L, Qiu J, Xing W (2018) CT-based radiomic model predicts high grade of clear cell renal cell carcinoma. Eur J Radiol 103:51-56.

doi:10.1016/j.ejrad.2018.04.013

50. Nazari M, Shiri I, Hajianfar G, Oveisi N, Abdollahi H, Deevband MR, Oveisi M, Zaidi H (2020) Noninvasive Fuhrman grading of clear cell renal cell carcinoma using computed tomography radiomic features and machine learning. Radiol Med 125 (8):754-762.

doi:10.1007/s11547-020-01169-z 
51. Bektas CT, Kocak B, Yardimci AH, Turkcanoglu MH, Yucetas U, Koca SB, Erdim C, Kilickesmez O (2019) Clear Cell Renal Cell Carcinoma: Machine Learning-Based Quantitative Computed Tomography Texture Analysis for Prediction of Fuhrman Nuclear Grade. Eur Radiol 29 (3):1153-1163. doi:10.1007/s00330-018-5698-2

52. Lin F, Ma C, Xu J, Lei Y, Li Q, Lan Y, Sun M, Long W, Cui E (2020) A CT-based deep learning model for predicting the nuclear grade of clear cell renal cell carcinoma. Eur J Radiol 129:109079. doi:10.1016/j.ejrad.2020.109079

53. Lee HW, Cho HH, Joung JG, Jeon HG, Jeong BC, Jeon SS, Lee HM, Nam DH, Park WY, Kim CK, Seo SI, Park H (2020) Integrative Radiogenomics Approach for Risk Assessment of PostOperative Metastasis in Pathological T1 Renal Cell Carcinoma: A Pilot Retrospective Cohort Study. Cancers (Basel) 12 (4). doi:10.3390/cancers12040866

54. Feng Z, Zhang L, Qi Z, Shen Q, Hu Z, Chen F (2020) Identifying BAP1 Mutations in ClearCell Renal Cell Carcinoma by CT Radiomics: Preliminary Findings. Front Oncol 10:279. doi:10.3389/fonc.2020.00279

55. Kocak B, Durmaz ES, Kaya OK, Kilickesmez O (2020) Machine learning-based unenhanced CT texture analysis for predicting BAP1 mutation status of clear cell renal cell carcinomas. Acta Radiol 61 (6):856-864. doi:10.1177/0284185119881742

56. Kocak B, Durmaz ES, Ates E, Ulusan MB (2019) Radiogenomics in Clear Cell Renal Cell Carcinoma: Machine Learning-Based High-Dimensional Quantitative CT Texture Analysis in Predicting PBRM1 Mutation Status. AJR Am J Roentgenol 212 (3):W55-w63.

doi:10.2214/ajr.18.20443

57. Chen X, Zhou Z, Hannan R, Thomas K, Pedrosa I, Kapur P, Brugarolas J, Mou X, Wang J (2018) Reliable gene mutation prediction in clear cell renal cell carcinoma through multiclassifier multi-objective radiogenomics model. Phys Med Biol 63 (21):215008.

doi:10.1088/1361-6560/aae5cd

58. Ghosh P, Tamboli P, Vikram R, Rao A (2015) Imaging-genomic pipeline for identifying gene mutations using three-dimensional intra-tumor heterogeneity features. J Med Imaging (Bellingham) 2 (4):041009. doi:10.1117/1.Jmi.2.4.041009

59. Corwin MT, Altinmakas E, Asch D, Bishop KA, Boge M, Curci NE, Ebada M, Elkassem AA, Fananapazir G, Fetzer DT, Gaballah AH, Gandhi D, Kampalath R, Lee S, Markese M, Mclnnes MD, Patel NU, Remer EM, Rosasco S, Schieda N, Sweet DE, Smith AD, Taylor E, Silverman SG, Davenport MS (2020) Clinical importance of incidental homogeneous renal masses 10-40 $\mathrm{mm}$ and 21-39 Hounsfield Units at portal venous-phase CT: A 12-institution retrospective cohort study. AJR Am J Roentgenol. doi:10.2214/ajr.20.24245

60. Kocak B, Kaya OK, Erdim C, Kus EA, Kilickesmez O (2020) Artificial Intelligence in Renal Mass Characterization: A Systematic Review of Methodologic Items Related to Modeling, Performance Evaluation, Clinical Utility, and Transparency. AJR Am J Roentgenol:1-10. doi:10.2214/ajr.20.22847

61. Kocak B, Durmaz ES, Erdim C, Ates E, Kaya OK, Kilickesmez O (2020) Radiomics of Renal Masses: Systematic Review of Reproducibility and Validation Strategies. AJR Am J Roentgenol 214 (1):129-136. doi:10.2214/ajr.19.21709 